\title{
EchoGéo
}

$51 \mid 2020$

Territoires « cyclonés »

\section{Territoires « cyclonés ». Les aléas cycloniques et leurs impacts}

Introduction

\section{Freddy Vinet et Frédéric Leone}

\section{OpenEdition}

Journals

\section{Édition électronique}

URL : https://journals.openedition.org/echogeo/18621

DOI : 10.4000/echogeo.18621

ISSN : 1963-1197

\section{Éditeur}

Pôle de recherche pour l'organisation et la diffusion de l'information géographique (CNRS UMR 8586)

\section{Référence électronique}

Freddy Vinet et Frédéric Leone, "Territoires " cyclonés ». Les aléas cycloniques et leurs impacts », EchoGéo [En ligne], 51 | 2020, mis en ligne le 28 janvier 2021, consulté le 10 août 2021. URL : http:// journals.openedition.org/echogeo/18621 ; DOI : https://doi.org/10.4000/echogeo.18621

Ce document a été généré automatiquement le 10 août 2021.

EchoGéo est mis à disposition selon les termes de la licence Creative Commons Attribution - Pas d'Utilisation Commerciale - Pas de Modification 4.0 International (CC BY-NC-ND) 


\title{
Territoires « cyclonés ». Les aléas cycloniques et leurs impacts
}

\author{
Introduction
}

\author{
Freddy Vinet et Frédéric Leone
}

1 Ce dossier d'EchoGéo est centré sur les conséquences environnementales, sociales, économiques et politiques des cyclones sur les territoires, et leur gestion. Le néologisme « cycloné » est apparu sur les réseaux sociaux en 2017 après le passage du cyclone Irma aux Antilles. Il renvoie à tout type de destruction causée par des cyclones de catégorie 4 ou 5, mais aussi à l'expérience humaine éprouvante vécue par les sinistrés. Ces événements posent question en termes d'évaluation ex ante et ex post des dommages et en termes de gestion: prévision, préparation, gestion de crise, reconstruction; le tout dans une optique d'atténuation des impacts. Leur analyse spatiale et multi-dimensionnelle contribue au développement d'une géographie mondiale des risques et des catastrophes, en plein essor depuis une vingtaine d'année dans nos universités françaises. Elle démontre une fois de plus les capacités d'analyse, d'adaptation et de réponse de notre discipline face aux problématiques socioenvironnementales actuelles, à haut risque pour nos sociétés et nos territoires.

2 Les dernières années ont vu l'occurrence de cyclones particulièrement puissants comme Haiyan aux Philippines en 2013 qui aurait battu le record de basse pression, ou Irma et Maria aux Antilles, deux ouragans successifs qui ont atteint la catégorie 5 en septembre 2017 à seulement dix jours d'intervalle. Depuis 1851, les centres d'observation ont recensé 381 cyclones tropicaux majeurs qui ont atteint ou dépassé la catégorie 4 de l'échelle de Saffir-Simpson (source IBTrACS/NOAA, 2020), principalement dans les bassins de l'Atlantique Nord et du Nord-Ouest du Pacifique (illustration 1). Les bilans humains mondiaux font état de 450000 décès depuis 1975 (1419 cyclones meurtriers), avec des événements particulièrement dramatiques en Inde en 1977, au Bangladesh en 1985 et 1991, au Honduras en 1998 ou au Myanmar en 2008 ... (source EMDAT/CRED, 2020). Les lourds bilans humains ne sont d'ailleurs pas l'apanage des pays pauvres comme a permis d'en juger le cas de Katrina qui fit 1833 victimes au Sud des États-Unis en 2005 (Knabb et al., 2007). Ces dépressions 
tourbillonnaires et leur cortège d'aléas induits (inondations, mouvements de terrain, submersion marine) ont provoqué des pertes économiques considérables, estimées à 2128 milliards de dollars US à travers le monde depuis 1980 (source NatCat Service/ Munich RE, 2020). S'ajoutent à cela les impacts non monétisables comme les effets environnementaux, en particulier sur les écosystèmes et morpho-systèmes côtiers (Wang et Horwitz, 2007 ; Zhang et al., 2012), ou les conséquences sanitaires ou sociales (Rappaport, 2014). La concentration spatiale des enjeux sur les littoraux suscite les inquiétudes des assureurs et des réassureurs qui voient ces charges de sinistres augmenter. La dévastation des petits ensembles insulaires (habitats, infrastructures et installations touristiques détruits, communications coupées, etc.) met en exergue les causes profondes des vulnérabilités de ces territoires : isolement géographique, exiguïté, disparités économiques, ségrégation socio-spatiale, forte exposition du littoral, manque de ressources locales, problèmes de gouvernance... Le passage de l'ouragan Irma dans la Caraïbe en septembre 2017 nous en a apporté une belle démonstration.

Illustration 1 - L'activité cyclonique et son bilan humain. Territoires étudiés dans ce numéro spécial.

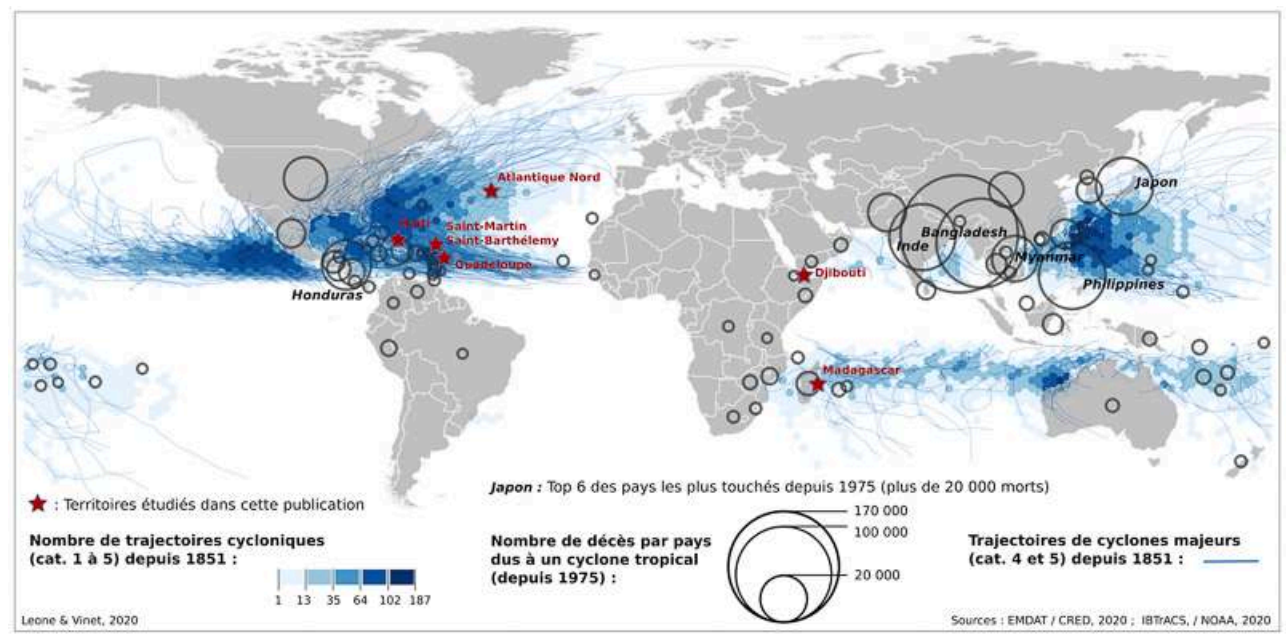

D'après les prévisions du GIEC, les cyclones ne seraient pas à l'avenir plus nombreux mais plus puissants même si le passé récent ne corrobore pas toujours cette tendance notamment dans le Pacifique ouest (Hoarau et al., 2018). Se pose également la question de l'élargissement en latitude de la zone concernée par ces phénomènes (Pilon et al., 2017). Les méthodes de quantification et de modélisation a priori et a posteriori se développent considérablement notamment par l'utilisation de l'imagerie satellite et aéroportée légère (Fernandez-Galarreta et al., 2015). Comment prévoir les dégâts d'un potentiel cyclone? Peut-on les catégoriser, les quantifier, les cartographier ex ante? Après le passage d'un cyclone, les outils de quantification des impacts économiques des cyclones fournissent quasiment en temps réel des éléments d'aide à la décision appréciables pour les décideurs et les gestionnaires des territoires. Les défis à relever et à anticiper pour faciliter le relèvement des territoires cyclonés sont nombreux: relogement des sinistrés, remise en état des réseaux et des services publics (éducation), gestion des déchets, pollutions diverses, risque sanitaire. Leur meilleure gestion passe par une planification préalable (plan de continuité d'activité) et un renforcement des capacités des sociétés exposées (Gaillard et al., 2010). 
4 En termes de gestion de crise, comment anticiper au mieux les événements? L'adaptation des réponses institutionnelles aux spécificités locales est un point clé de la gestion de crise au travers de problèmes tels que l'évacuation préventive des zones touchées ou les dysfonctionnements dans la communication (entre gestionnaires de crise, entre autorités et public...). Comment les expériences passées sont-elles réinvesties dans la gestion des crises cycloniques? Y a-t-il capitalisation des expériences ou bien chaque cas étant différent, la gestion de crise prend-elle à chaque fois une grande part d'improvisation?

5 Au-delà des retours d'expérience « à chaud ", ces phénomènes intenses doivent se lire à l'aune de leurs conséquences à long terme sur les territoires. Ces événements sont-ils des ruptures, des bifurcations dans les trajectoires de ces territoires ou bien ne remettent-ils pas en cause le fonctionnement à long terme de leurs modèles de développement? Décideurs et scientifiques ont besoin de retours d'expérience sur le long terme, sur les impacts mais aussi sur les phases de reconstruction (Moatty et al., 2017). Le relèvement a-t-il été efficace préventivement, rapide dans ses ressorts économiques, éthique sur le plan de la justice sociale? Quels changements physiques et sociétaux a induit le passage du cyclone? La conscience du risque et sa prévention se sont-elles améliorées? La phase de reconstruction s'avère primordiale (Ingram et al. 2006). Elle est en théorie une "fenêtre d'opportunité» (Christoplos, 2006) pour développer la résilience territoriale en cas de futurs événements mais elle fait apparaitre des différences criantes entre des territoires durablement impactés (Kelman, 2014) et d'autres capables de se redresser promptement. Le relèvement rapide de l'archipel des Vanuatu (Pacifique Ouest) après le cyclone Pam en 2015 a montré la résilience de sociétés traditionnelles pourtant fortement exposées (Rey et al., 2017 : Le Dé et al., 2018). Enfin, la compréhension des relations cyclone/territoire ne peut faire l'économie d'une mise en perspective de ces catastrophes dans la trajectoire de vulnérabilité globale des territoires (Duvat et al., 2017 ; Desarthe et Moncoulon, 2017).

6 Ce dossier d'EchoGéo éclaire plusieurs de ces aspects en privilégiant une approche géographique. Il rassemble treize contributions, portant sur sept territoires cyclonés, depuis les Petites Antilles du Nord (Saint-Martin, Saint-Barthélemy) et l'Atlantique Nord, en passant par la Guadeloupe et Haïti, jusqu'à Djibouti et Madagascar dans l'Océan Indien (illustration 1). Sept de ces articles s'attardent en particulier sur la saison cyclonique 2017, particulièrement éprouvante pour les Antilles françaises.

Une première série de quatre articles aborde la question des impacts, thème central de ce numéro. Thibault Catry, Christophe Révillion, Pascal Mouquet, et Gwenaëlle Pennober montrent l'apport de l'imagerie satellite pour le suivi de l'impact des événements cycloniques à Madagascar, en jouant sur la complémentarité des échelles et des capteurs et en prenant pour cas d'étude deux cyclones (Haruna en 2013 et Enawo en 2017). Tony Rey, Thomas Candela, Matthieu Péroche et Frédéric Leone mobilisent de leur côté l'approche de terrain et l'imagerie par drone pour évaluer les changements côtiers et les inondations consécutifs au passage de l'ouragan Irma des 5 et 6 septembre 2017 sur les îles de Saint-Martin et de Saint-Barthélemy. Jean-Philippe Cherel, Mustapha Nour Ayeh, Bouh Omar Ali et Freddy Vinet analysent un événement assez singulier (le cyclone Sagar) survenu en mai 2018 dans le golfe d'Aden, et ses conséquences socio-économiques sur la ville de Djibouti peu accoutumée à ce genre d'événement. Frédéric Leone, Samuel Battut, Victoria Bigot, Guilhem Cousin Thorez, Thomas Candela et Freddy Vinet posent les bases méthodologiques d'un catalogue 
historique des événements hydro-météorologiques significatifs et mortels qui ont frappé l'archipel de Guadeloupe et les îles du Nord depuis 1635, offrant ainsi la possibilité de comprendre les principaux facteurs de vulnérabilité humaine face aux cyclones et leur évolution au cours du temps.

Deux autres articles traitent de la crise cyclonique et de sa gestion, d'un point de vue institutionnel ou individuel. Valérie November, Alice Azémar, Sophie Lecacheux et Thierry Winter analysent les obstacles à une « bonne » gestion de crise et soulignent la fragmentation du couple anticipation/décision dans la pratique des différents niveaux de réponse, en décortiquant deux études de cas situées pendant la crise Irma-JoséMaria de 2017 aux Antilles, l'une observée au niveau interministériel, et l'autre vécue par un opérateur de l'État, au niveau territorial. Stéphanie Defossez et Monique Gherardi ciblent quant à elles, et pour le même évènement, le rôle des populations dans la gestion de la crise à Saint-Martin, en privilégiant un suivi par entretien des personnes à plusieurs échéances après le cyclone.

9 Les trois contributions qui suivent reviennent sur les problématiques post-crise de relèvement et de reconstruction des territoires cylonés. Annabelle Moatty, Delphine Grancher, Clément Virmoux et Julien Cavero abordent la question du relèvement après le cyclone Irma à Saint-Martin à travers le vécu et la perception des adolescents, en considérant les récits des adolescents en tant que données susceptibles d'alimenter les retours d'expérience post-catastrophe. Toujours pour le même événement et SaintMartin, Marie Cherchelay montre le processus de reconstruction par le prisme du tourisme, afin d'analyser les jeux d'acteurs complexes qui se développent, autour des enjeux et attentes qui émergent en période post-crise. Simon Veitl interroge de son côté le rôle des interventions humanitaires internationales pour la reconstruction en Haïti, en particulier au niveau des écoles du département Sud, après l'ouragan Matthew survenu en 2016.

Une dernière série de quatre articles pose la question des évolutions et des adaptations face au risque cyclonique à long terme. L'historien Jérémy Desarthe revient sur l'ouragan majeur de septembre 1928 en Guadeloupe et sur ses conséquences, traçables jusqu'en 1934 grâce à une exploitation des archives administratives. Karl Hoarau, Florence Pirard et Ludovic Chalonge analysent l'influence du réchauffement climatique actuel sur l'activité des ouragans extrêmes de l'Atlantique Nord (1945-2018), dont l'intensité et la fréquence sont estimées grâce aux reconnaissances aériennes et à la technique de Dvorak fondée sur la réinterprétation d'images satellitaires. La question du temps long des sociétés « cyclonées » est ensuite abordée par Fanny Benitez, Magali Reghezza-Zitt et Nancy Meschinet de Richemond qui interrogent l'utilisation de la notion de « culture du risque " par les différents acteurs du champ de la prévention des catastrophes naturelles et de la gestion de crise en Guadeloupe, en particulier à l'aune de la saison cyclonique 2017. Enfin, Michel Desse, Monique Gherardi et Simon Charrier montrent en quoi les cyclones engendrent des ruptures positives ou négatives pour les sociétés et les économies insulaires de Guadeloupe, très dépendantes de leurs littoraux et des activités touristiques qui les habitent. 


\section{BIBLIOGRAPHIE}

Blaikie P., Cannon T., Davis I., Wisner B., 1994. At risk: natural hazards, people's vulnerability, and disasters. Londres, Ed. Routledge.

Christoplos I., 2006. The elusive "window of opportunity" for risk reduction in post-disaster recovery, Intervention. ProVention Consortium Forum, Bangkok, 2-3 Février 2006, 4 p.

Desarthe J., Moncoulon D., 2017. Quatre siècles de cyclones tropicaux dans les départements d'outre-mer. La Météorologie, $\mathrm{n}^{\circ} 99$.

Duvat V., Magnan A., Russell M. et al., 2017. Trajectories of exposure and vulnerability of small islands to climate change. WIREs Clim Change [En ligne], e478. DOI: https://doi.org/10.1002/wcc. 478

Fernandez-Galarreta J., Kerle N., Gerke M., 2015. UAV based urban structural damage assessment using object based image analysis and semantic reasoning. Nat. Hazards Earth Syst. Sci., vol. 15, p. 1087-1101.

Gaillard J.-C., Wisner B., Benouar D. et al., 2010. Alternatives pour une réduction durable des risques de catastrophe. Human Geography, vol. 3, n 1, p. 66-88.

Hoarau K., Chalonge L., Pirard F., Peyrusaubes D. 2018. Extreme tropical cyclone activities in the southern Pacific Ocean. International Journal of Climatology, vol. 38, p. 1409-1420.

Ingram J. C., Franco G., Rumbaitis-Del Rio, C., Khazai B., 2006. Post-disaster recovery dilemmas: challenges in balancing short-term and long-term needs for vulnerability reduction. Environmental Science \& Policy, vol. 9, p. 607-613.

Kelman I., 2014. Climate Change and Other Catastrophes: Lessons from Island Vulnerability and Resilience. Moving Worlds: a journal for transcultural writings, vol. 14, n² 2, p. 127-140.

Knabb R. D., Rhome J. R., Brown D. P., 2007. National Hurricane Center. Tropical cyclone report: Hurricane Katrina, August 23-30, 2005. Miami, National Oceanic and Atmospheric Administration, National Weather Service, National Hurricane Center. https://www.nhc.noaa.gov/data/tcr/ AL122005_Katrina.pdf consulté le 15 Avril 2020.

Le Dé L., Rey T., Leone F., David G., 2018. Sustainable livelihoods and efFectiveness of disaster responses: a case study of tropical cyclone Pam in Vanuatu, Naturals Hazards, vol. 91, $\mathrm{n}^{\circ} 3$, p. 1203-1221.

Moatty A., Gaillard J.C., Vinet F., 2017. Du désastre au développement : Les enjeux de la reconstruction post-catastrophe. Annales de géographie, vol. 714, nº 2, p. 169-194.

Pilon R., Chauvin F., Belmadani A., Palany P., 2017. North Atantic tropical cyclones: Historical simulations and future changes with the new high-resolution Arpege AGCM. AGU Fall Meeting, paper A53C-2258. URL: https://agu.confex.com/agu/fm17/meetingapp.cgi/Paper/239137

Rappaport E., 2014. Fatalities in the United States from Atlantic Tropical Cyclones: New Data and Interpretation. Bull. Am. Met. Soc. [En ligne], may. DOI: https://doi.org/10.1175/BAMS-

D-12-00074.1

Rey T., Le Dé L., Leone F., David G., 2017. An integrative approach to understand vulnerability and resilience postdisaster: the 2015 cyclone PAM in urban Vanuatu as case study, Disaster Prevention Management: an International Journal [En ligne], vol. 26, n 3, p. 259-275. DOI: https://doi.org/ 10.1108/DPM-07-2016-0137 
Wang P., Horwitz M. H., 2007. Erosional and depositional characteristics of regional overwash deposits caused by multiple hurricanes. Sedimentology [En ligne], vol. 54, p. 545-564. DOI : https:// doi.org/10.1111/j.1365-3091.2006.00848.x

Zhang K., Liu H., Li Y., Smith T.J., 2012. The role of mangroves in attenuating storm surges. Estuarine, Coastal and Shelf Science [En ligne], vol. 102-103, p. 11-23. DOI: https://doi.org/10.1016/ j.ecss.2012.02.021

INDEX

Thèmes : Sur le Champ - Sur le Terrain

\section{AUTEURS}

\section{FREDDY VINET}

Freddy Vinet, freddy.vinet@univ-montp3.fr, est professeur de géographie des risques naturels à l'Université Paul-Valéry Montpellier 3. Il a récemment publié :

- Vinet F., 2018. La Grande Grippe. 1918 : la pire épidémie du siècle. Histoire de la grippe espagnole. Paris, Éd. Vendémiaire, $260 \mathrm{p}$.

- Vinet F. (dir.), 2018. Inondations 1. La connaissance du risque. Londres, Elsevier/ISTE éditions, $388 \mathrm{p}$.

- Vinet F. (dir.) 2018. Inondations 2. La gestion du risque. Londres, Elsevier/ISTE éditions, 470 p.

\section{FRÉDÉRIC LEONE}

Frédéric Leone, frederic.leone@univ-montp3.fr, est professeur de géographie des risques naturels à l'Université Paul-Valéry Montpellier 3. Il a récemment publié :

- Rey T., Leone F., Candela T. et al., 2019. Coastal Processes and Influence on Damage to Urban Structures during Hurricane Irma (St-Martin \& St-Barthélemy, French West Indies). Journal of Marine Science and Engineering [En ligne]. vol. 7, n 7, p. 215. DOI: https://doi.org/10.3390/ jmse7070215

- Bennani O, Druon E., Leone F. et al., 2019. A spatial and integrated flood risk diagnosis. Relevance for disaster prevention at Ourika valley (High Atlas-Morocco). Disaster Prevention and Management [En ligne], vol. 28 nº 5, p. 548-564. DOI : https://doi.org/10.1108/DPM-12-2018-0379 - Leone F., Komorowski J.C., Gherardi-Leone M. et al., 2018. Accessibilité territoriale et gestion de crise volcanique aux Antilles françaises (Guadeloupe \& Martinique) : contribution à la planification des évacuations. Cybergeo. European Journal of Geography, Espace, Société, Territoire [En ligne], document 865. http://journals.openedition.org/cybergeo/29425 - DOI: https://doi.org/ 10.4000/cybergeo.29425Fr25/04/2020 